EPJ Web of Conferences 70, 00058 (2014)

DOI: $10.1051 /$ epjconf/ 20147000058

(C) Owned by the authors, published by EDP Sciences, 2014

\title{
Heavy Flavour Production at LHC: an overview
}

\author{
Giuseppe Eugenio Bruno ${ }^{1, a}$ \\ ${ }^{1}$ Dipartimento Interateneo di Fisica 'M. Merlin' and Sezione INFN, Bari, Italy
}

\begin{abstract}
An overview of experimental results on the production of heavy flavour (charm and bottom) hadrons at LHC is presented. Both the open and hidden heavy flavour sectors are covered, with an emphasis on heavy ion collisions.
\end{abstract}

\section{Introduction}

The measurement of the production of hadrons with open heavy flavour (HF) in pp collisions at the LHC provides a way to test, in a new energy domain, calculations of QCD processes based on the factorization approach. In this scheme, the cross sections are computed as a convolution of the parton distribution functions of the incoming protons, the partonic hard scattering cross sections, and the fragmentation functions. Recent implementations of such calculations, at the perturbation level of next-to-leading order or at fixed order with next-to-leading-log resummation (FONLL) [1] describe well the beauty production cross section measured in $\mathrm{p} \overline{\mathrm{p}}$ collisions at $\sqrt{s}=1.96 \mathrm{TeV}$ at the FNAL Tevatron collider [2-4]. The production cross section of charmed hadrons (D mesons) at Tevatron is reproduced within the theoretical uncertainties of the calculations as well [5-7]. However, the comparison suggests that charm production is underestimated by the results obtained with the central values of the calculation parameters, as observed also in pp collisions at the BNL RHIC collider at the lower energy of $\sqrt{s}=200 \mathrm{GeV}[8,9]$. In this context, it is particularly interesting to perform the comparison for charm and beauty production at the LHC energy, which is more than three times higher than at the Tevatron. Furthermore, at LHC energies, the measurement of charm production in the low transverse momentum $\left(p_{\mathrm{T}}\right)$ region probes the parton distribution functions of the proton at small values of parton fractional momentum $x$ and squared momentum transfer $Q^{2}$. For illustration, using a simplified $2 \rightarrow 2$ kinematics at leading order, c quarks $\left(m_{\mathrm{c}} \approx 1.5 \mathrm{GeV} / c^{2}\right)$ with $p_{\mathrm{T}} \sim 2 \mathrm{GeV} / c$ and rapidity $y \sim 0$ probe the parton distribution functions at $x \sim 7 \times 10^{-4}$ and $Q^{2} \sim(5 \mathrm{GeV})^{2}$, where the gluon component is dominant. In this kinematic regime, the gluon distribution may reach the level of saturation, leading to a measurable departure of the observed cross sections from the expectations based on the factorization approach (see e.g. [1]).

In heavy-ion interactions the momentum distributions of charm and beauty hadrons is influenced by the formation of hot and dense QCD matter. The effect is quantified using the nuclear modification factor:

$$
R_{\mathrm{AA}}=\frac{\mathrm{d} N /\left.\mathrm{dp}_{\mathrm{T}}\right|_{\mathrm{Pb}-\mathrm{Pb}}}{<N_{\text {coll }}>\mathrm{d} N /\left.\mathrm{dp}_{\mathrm{T}}\right|_{\mathrm{pp}}}
$$

\footnotetext{
ae-mail: giuseppe.bruno@ba.infn.it
} 
Heavy-quarks crossing through the QCD matter interact with the medium and may lose energy via various mechanisms. The radiative energy loss of a hard parton (quark or gluon) is expected to be proportional to the QCD Casimir coupling to other gluons from the medium [1]. Light and heavy quarks should then lose less energy than gluons due to their color charge. In addition, due to their relative large mass, heavy-quarks gluon-bremsstrahlung should be suppressed at large angles as a consequence of destructive quantum interferences. Hence a hierarchy is anticipated for $R_{\mathrm{AA}}$, when going from the mostly gluon-originated light-flavour hadrons (e.g., pions) to $\mathrm{D}$ and to $\mathrm{B}$ mesons: $R_{\mathrm{AA}}^{\pi}<R_{\mathrm{AA}}^{\mathrm{D}}<R_{\mathrm{AA}}^{\mathrm{B}}$. RHIC results show that heavy-quarks lose energy in the medium, but a possible quark-mass hierarchy has not yet been elucidated.

The mechanisms of heavy quarkonium (charmonium and bottonomium) production operate at the boundary of the perturbative and non-perturbative regimes of QCD. At hadron colliders, J/ $\psi$ production was extensively studied at the Tevatron $[1,1,1,1]$ and RHIC [1], along with other charmonuim states and the $\Upsilon$ family. However, the underlying production mechanism is still not well understood. Several models exist but fail to reproduce both the cross-section and the polarization measurements at the Tevatron. Among these are the Colour Singlet Model (CSM) [1, 1, 1], recently improved by adding higher order contributions (NLO CSM), the Colour Evaporation Model (CEM) [2], and the non-perturbative Colour Octet Mechanism (COM) [2, 2, 2], which is studied in the framework of nonrelativistic QCD (NRQCD). Measurements in the new energy domain of the Large Hadron Collider (LHC) can contribute to a deeper understanding of the physics of the hadroproduction processes.

The suppression of heavy quarkonium states in heavy-ion collisions was proposed long ago [2] as a clean signature of a formation of a Quark-Gluon Plasma (QGP). In addition to the color screening mechanism proposed in [2] as the main source of suppression, it became soon clear that other effects may contribute, as dissociation in cold nuclear matter [2] and/or in the hot confined medium [2]. Also initial state effects, such as nuclear shadowing [2] and initial state parton energy loss [2] are expected to play a role. On the experimental side, lots of data are now available at SPS [2, 3] and RHIC [3,3] energies, and a heavy quarkonium (QQ̄) suppression beyond cold nuclear matter effects (the so-called "anomalous suppression") was detected, either by studying the ratio $\sigma_{\mathrm{QQ}} / \sigma_{\text {Drell-Yan }}$ or the nuclear modification factor $R_{\mathrm{AA}}$, for different collision systems and as a function of the centrality and of the quarkonium kinematic variables [3] . It is commonly accepted that the observed anomalous suppression is likely to be related to the formation of a deconfined state in nuclear collisions. At LHC, due to large c̄ multiplicity, a new mechanism for the $\mathrm{J} / \psi$ production can take place: partonic transport models [3,3], as well as statistical generation models [3], predict a reduced $\mathrm{J} / \psi$ suppression (or even an enhanced one) as being due to a (re)generation of $\mathrm{J} / \psi$ along the collision history and/or at hadronization.

Non-central nucleus-nucleus collisions present a geometrical anisotropy with respect to the reaction plane of the collision (the plane defined by the beam direction and the impact parameter vector ${ }^{1}$ ). This initial spatial anisotropy can evolve into a momentum anisotropy of produced low $p_{\mathrm{T}}$ particles driven by different pressure gradients in the in-plane (along the impact parameter) and out-of-plane (perpendicular to the impact parameter vector) directions. The momentum anisotropy can be then quantified via a Fourier expansion of the azimuthal angle with respect to the event plane. The second coefficient of this expansion $v_{2}$ is called elliptic flow. The measurement of heavy-flavour particles $v_{2}$ (for both open and hidden $\mathrm{HF}$ ), in combination to the nuclear modification factor $R_{\mathrm{AA}}$, allows to probe the degree of thermalization of the medium, the heavy flavour hadronization mechanism and the path length dependence of the heavy-quark energy loss [3, 3].

\footnotetext{
${ }^{1}$ The impact parameter is the vector, in the transverse plane, from the centre of the target nucleus to that of the projectile.
} 
At the Large Hadron Collider (LHC) the study of heavy flavour production in hadronic collisions has entered into the precision era. In fact, the production cross sections of $c \bar{c}$ and $b \bar{b}$ quark pairs increase very steeply with the center-of-mass energy of the colliding pp or $\mathrm{p} \overline{\mathrm{p}}$ system, see Fig. 1.
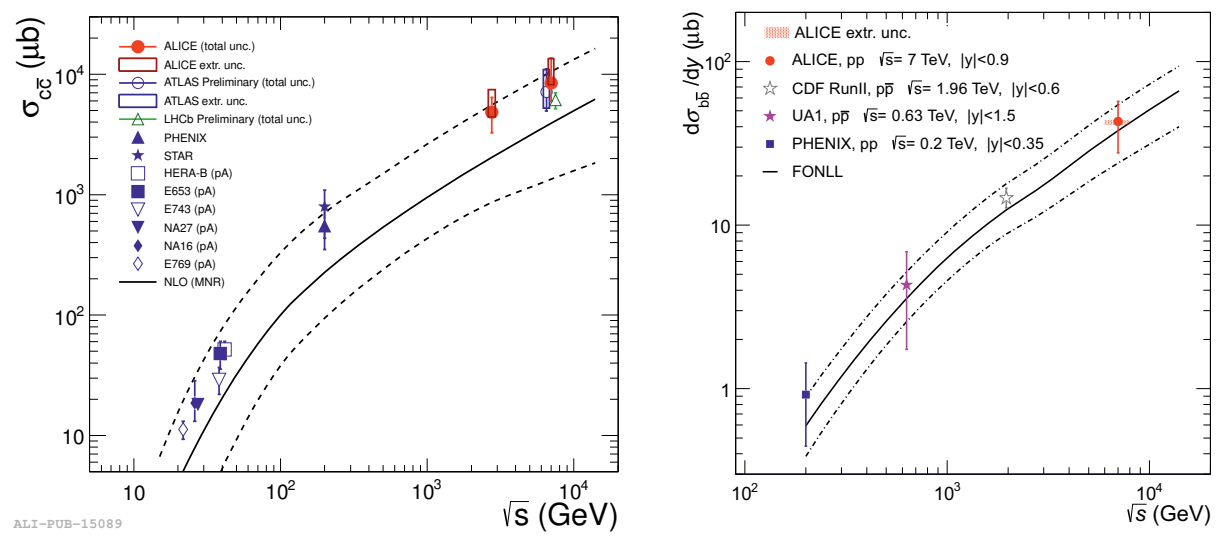

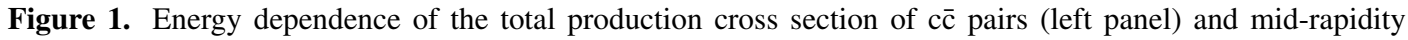
$\mathrm{d} \sigma_{\mathrm{b} \bar{b}} / \mathrm{dy}$ (right panel), see [3,4] and references therein. In case of proton-nucleus (pA) or deuteron-nucleus (dA) collisions, the measured cross sections have been scaled down by the number of binary nucleon-nucleon collisions calculated in a Glauber model of the proton-nucleus or deuteron-nucleus collision geometry. The pQCD NLO calculations (and their uncertainties) are represented by solid (dashed) lines.

The four main experiments at LHC, namely ALICE [4], ATLAS [4], CMS [4] and LHCb [4], can study heavy flavour production with complementary capabilities: high $p_{\mathrm{T}}$, high rates and full jet reconstruction, over extended rapidity $(y)$ range, are the strengh of ATLAS and CMS. LHCb is the dedicated $\mathrm{HF}$ experiment at the LHC, but it does not have a heavy ion program. ALICE, the dedicated heavy ion experiment, complements the capabilities of the other experiments in pp collisions, thanks to its reduced material budget, lower magnetic field and excellent particle identification.

\section{2 pp collisions}

\subsection{Open heavy flavour}

Charmed mesons have been studied in exclusive decays into charged hadrons (i.e. $\mathrm{D}^{0} \rightarrow \mathrm{K}^{-} \pi^{+}$, $\mathrm{D}^{+} \rightarrow \mathrm{K}^{-} \pi^{+} \pi^{+}$and $\mathrm{D}^{*+} \rightarrow \mathrm{D}^{0} \pi^{+}$) at central rapidity by ALICE [4] down to $p_{\mathrm{T}}=1 \mathrm{GeV} / c$ of the reconstructed meson. Preliminary results have been reported also by ATLAS (at mid-rapidity) and $\mathrm{LHCb}$ (at forward rapidity) experiments. This is undoubtedly the best way to study charm production. It can also be studied, more indirectly, by considering the semi-leptonic decays of HF hadrons into leptons (e or $\mu$ ) $[4,4,4]$. 


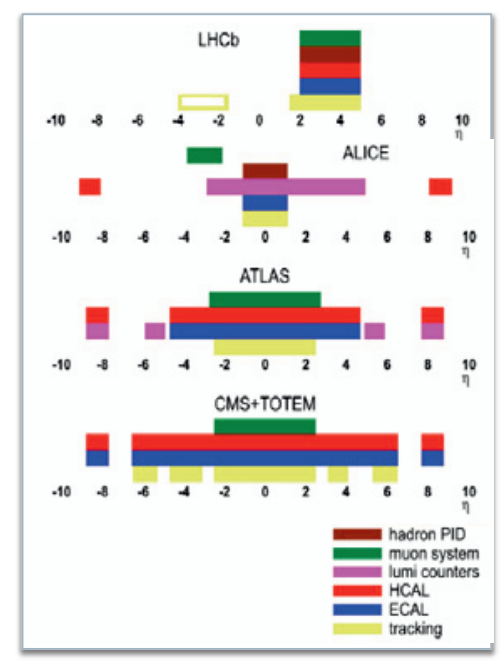

Figure 2. Schematic drawing of the pseudo-rapidity coverage of the four LHC main experiments for different detection capabilities.

The LHC experiments have reported measurements of b-hadron production in pp collisions at $\sqrt{s}=7 \mathrm{TeV}$ by studying either exclusive decays of B mesons [4, 5, 5] or semi-inclusive decays of b-hadrons $[4,5,5,5,5,5,5]$.

All measured cross sections are reproduced within uncertainties by theoretical predictions based on perturbative QCD at fixed order with next-to leading-log (FONLL) $[1,5,5]$.

\subsection{Quarkonia production}

The first LHC results on the $\mathrm{J} / \psi$ transverse momentum $\left(p_{\mathrm{T}}\right)$ differential cross sections $[4,5,5,5,5,6]$ are well described by to next-to-leading order (NLO) non-relativistic QCD (NRQCD) theoretical calculations, which includes color-singlet (CS), color-octet $(\mathrm{CO})$ with both S-wave and ${ }^{3} P_{J}^{[8]}$ channels, and heavier charmonium feed-down contributions $[6,6]$. Similarly, the first measurements of $J / \psi$ polarization [6], which do not agree with NLO predictions for $\mathrm{J} / \psi$ polarization via the color-singlet (CS) channel $[6,6]$ and cannot even be explained by the contribution of the S-wave color-octet (CO) channels [6], are better described with new calculations which include also the contribution of the ${ }^{3} P_{J}^{[8]} \mathrm{CO}$ channels [6]. All LHC experiments have silicon micro-vertex detector and can separate the prompt from non-prompt (i.e. from beauty hadron decays) $\mathrm{J} / \psi$. Other charmonium states, namely $\chi_{c}$ and $\Psi(2 S)$, have been already studied with very good precision $[5,6]$, and their production is well described by NLO NRQCD models, as well.

Bottonomium cross section production (including the $\chi_{\mathrm{b}}$ ) have also been measured, both at central and forward rapidities $[6,7,7]$. At central rapidity, the cross-section of $\Upsilon(1 S)$ is about 3 times larger than that measured at the Tevatron. A comparison with theoretical NLO NRQCD models shows good agreement with the measured cross-sections of $\Upsilon(\mathrm{nS})$. However, the measurement of the differential cross-sections is not sufficient to discriminate amongst the various models, and studies of other observables such as the polarization are expected (preliminary results from CMS were in fact delivered at the time of the Conference). 


\subsection{New particles, decay channels and observables}

First new (HF) particles have been discovered at the LHC: orbitally-excited $\Lambda_{\mathrm{b}}^{0}$ baryons [7] and a new $\Xi_{\mathrm{b}}$ baryon with strong decay into $\Xi_{\mathrm{b}}^{-} \pi^{+}$(and c.c.) [7], which most likely corresponds to the $J^{P}=3 / 2^{+}$ companion of the $\Xi_{\mathrm{b}}$.

A new decay channel of the rare doubly heavy flavoured $\mathrm{B}_{\mathrm{c}}{ }^{+}$meson (into $\mathrm{J} / 4 \pi^{+} \pi^{-} \pi^{+}$) has been studied [7], its branching ratio (measured relative to that into $\mathrm{J} / \psi \pi^{+}$, the only one observed before) being found in agreement with theoretical predictions.

New observables are also being studied at the LHC. The most remarkable are the multiplicity dependence of the HF production in pp collisions [7], which has been discussed at this Conference in [7], and the double charm production. For the latter, in particular, the production of $\mathrm{J} / \psi$ mesons accompanied by open charm, and pairs of open charm hadrons has been reported by LHCb [7]. The predictions from gluon-gluon fusion [7, 7] are significantly smaller than the observed cross sections. Better agreement is found with the Double Parton Scattering (DPS) model $[8,8]$ if the effective cross section inferred from the Tevatron data is used. The absence of significant azimuthal or rapidity correlations provides support for this hypothesis. This interpretation is only partially supported by the $\mathrm{CC}$ data (e.g., $\mathrm{D}^{0} \mathrm{D}^{0}$ or $\mathrm{D}^{0} \mathrm{D}^{+}$): if DPS is assumed to dominate, the estimated effective cross section would be a factor 2-3 larger than in the $\mathrm{J} / \psi \mathrm{C}$ case. For $\mathrm{C} \overline{\mathrm{C}}$ events significant rapidity and azimuthal correlations are observed. These, as well as the invariant mass spectra for $\bar{C} \bar{C}$ events, suggest a sizeable contribution from the gluon splitting process to charm quark production.

\subsection{The reference for first heavy ion studies at LHC: pp at $2.76 \mathrm{TeV}$}

Data of excellent quality have been recorded by the four main LHC experiments at $7 \mathrm{TeV}$. For instance, integrated luminosities of about 5.6 and $5.7 \mathrm{fb}^{-1}$ were delivered, respectively, to ATLAS and CMS in the 2011 run. With about one thousandth of such statistics CMS could study in details the production of the $\Upsilon$ family [6], starting from the dimuon invariant mass distribution shown in the left panel of figure 3. Only a very short run, over few days of data taking, was instead dedicated to the pp collisions at $\sqrt{s}=2.76 \mathrm{TeV}$, which is the reference data sample for the study of the $\mathrm{Pb}-\mathrm{Pb}$ collisions. The corresponding dimuon invariant mass distributions measured by CMS $[8,8]$ is shown in the central panel of figure 3. This example reflects the status of all other HF studies: excellent data sets are available in pp at $\sqrt{s}=7 \mathrm{TeV}$ (and in Pb-Pb collisions at $\sqrt{s_{\mathrm{NN}}}=2.76$, as discussed later), low statistic is the limitation of the reference pp collisions at $\sqrt{s}=2.76 \mathrm{TeV}$. As a consequence, interpolation procedures are often elaborated in the analyses, which introduce large systematical errors.

\section{$3 \mathrm{~Pb}-\mathrm{Pb}$ collisions}

\subsection{Open heavy flavour}

At RHIC, the suppression of heavy-flavour hadrons, measured indirectly from their inclusive decay electrons $[8,8,8]$, was found to be compatible with that of pions and generally stronger than most expectations based on radiative energy loss [8]. The first measurement of the nuclear modification factor of $\mathrm{D}$ meson, based on the $\mathrm{Pb}-\mathrm{Pb}$ data sample collected in 2010, has been performed by ALICE [8], by reconstructing their weak decays into charged hadrons as it was done for pp collisions. The $\mathrm{D}^{0}$, $\mathrm{D}^{+}$, and $\mathrm{D}^{*+} R_{\mathrm{AA}}$, measured as a function of transverse momentum and centrality, is in the range $0.25-0.35$ for $5<p_{\mathrm{T}}<16 \mathrm{GeV} / c$ for the $20 \%$ most central collisions. For $p_{\mathrm{T}}$ below $5 \mathrm{GeV} / c$, and towards peripheral collisions, there is a tendency for an increase of $R_{\mathrm{AA}}$ for $\mathrm{D}^{0}$ mesons. The suppression is almost as large as that observed for charged particles, which are mainly light-flavour hadrons, 

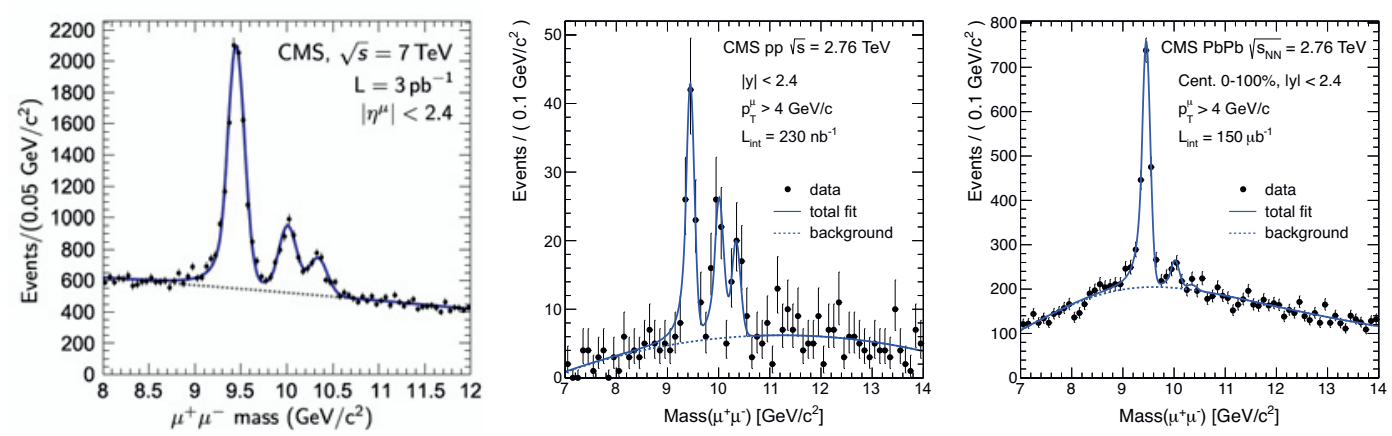

Figure 3. Dimuon invariant-mass distributions from the pp at $\sqrt{s}=7 \mathrm{TeV}$ (left), pp at $\sqrt{s}=2.76 \mathrm{TeV}$ (middle) and $2010 \mathrm{~Pb}-\mathrm{Pb}$ (right) data at $\sqrt{s_{\mathrm{NN}}}=2.76$, measured by the CMS experiment $[6,8]$.

with a possible indication, not fully significant with the present level of experimental uncertainties, of $R_{A A}^{\mathrm{D}}>R_{A A}^{\text {charged }}$. ALICE has also measured the inclusive production of muons from heavy-flavor decays at forward rapidity, $2.5<y<4$, as a function of $p_{\mathrm{T}}$ and collision centrality [8]. A weak suppression is measured in peripheral collisions. In the most central collisions, a suppression of a factor of about $3-4$ is observed in $6<p_{\mathrm{T}}<10 \mathrm{GeV} / c$. The suppression shows no significant $p_{\mathrm{T}}$ dependence.

The suppression of beauty hadrons has been studied for the first time by CMS considering the non-prompt $\mathrm{J} / \psi$ particles from $\mathrm{B}$ meson decays [9]. A strong suppression of non-prompt $\mathrm{J} / \psi$ mesons is observed in $\mathrm{Pb}-\mathrm{Pb}$ collisions when compared to pp collisions. This is the first unambiguous measurement of b-hadron suppression in heavy-ion collisions, which is likely connected to in-medium energy loss of $\mathrm{b}$ quarks. The average $p_{\mathrm{T}}$ of the non-prompt $\mathrm{J} / \psi$ in the measured kinematic range is $\approx 10 \mathrm{GeV} / c$, which translates into an average b-hadron $p_{\mathrm{T}}$ of $\approx 13 \mathrm{GeV} / c$.

The preliminary ALICE results, based on the much larger sample of $\mathrm{Pb}-\mathrm{Pb}$ collisions recorded in 2011, confirms the first results and they are discussed in [9]. Among these new results, the most remarkable is probably the first measurement the $\mathrm{D}$ meson elliptic flow. There are indications that $v_{2}>0$ in the $p_{\mathrm{T}}$ range $2<p_{\mathrm{T}}<6 \mathrm{GeV} / c$ with a $3 \sigma$ significance in the $30-50 \%$ centrality class. The result is also compatible with the $v_{2}$ of charged hadrons in the same $p_{\mathrm{T}}$ and rapidity region.

The overall emerging picture is that the expected hierarchy of $R_{\mathrm{AA}}$, which is discussed in section 1 , is observed, but of reduced size. This observation, together with the measured elliptic flow of D mesons, allows discriminating among the available theoretical models.

\subsection{Quarkonia production}

The differential $R_{\mathrm{AA}}$ results of $\mathrm{J} / \psi$ measured by ALICE down to zero transverse momentum are discussed in [7]. The main results include a suppression that saturates for semi-central to central events to values larger than the ones observed at RHIC. These results can be considered as strong hints for an important contribution of (re)generated $\mathrm{J} / \psi$ at low $p_{\mathrm{T}}$ in $\mathrm{Pb}-\mathrm{Pb}$ collisions at the LHC. An independent confirmation of this hypothesis may come from the study of the elliptic flow of $\mathrm{J} / \psi$. If (re)generation effects are sizeable, then the corresponding $\mathrm{J} / \psi$ would inherit the flow related to the collective expansion, which is experienced by the charm quarks contained in the fireball. Theoretical models predict in this case a non-zero $v_{2}$ for the $\mathrm{J} / \psi$ at intermediate $p_{\mathrm{T}}$ [9]. The preliminary results from ALICE provide a hint for a non-zero $\mathrm{v} 2$ signal at intermediate $p_{\mathrm{T}}$, which is in agreement with such a picture. 
The suppression of the individual $\Upsilon(\mathrm{nS})$ states in $\mathrm{PbPb}$ collisions with respect to their yield in $\mathrm{pp}$ data has been measured in details by the CMS experiment $[8,8]$. The results indicate a significant suppression of the $\Upsilon(\mathrm{nS})$ states in heavy-ion collisions compared to pp collisions at the same pernucleon-pair energy. The data support the hypothesis of increased suppression of less strongly bound states: the $\Upsilon(1 \mathrm{~S})$ is the least suppressed and the $\Upsilon(3 \mathrm{~S})$ is the most suppressed of the three states. The $\Upsilon(1 \mathrm{~S})$ and $\Upsilon(2 \mathrm{~S})$ suppressions are observed to increase with collision centrality. The suppression of $\Upsilon(2 S)$ is stronger than that of $\Upsilon(1 S)$ in all centrality ranges, including the most peripheral bin. The observed $\Upsilon(\mathrm{nS})$ yields contain contributions from decays of heavier bottomonium states and, thus, the measured suppression is affected by the dissociation of these states. This feed-down contribution to the $\Upsilon(1 \mathrm{~S})$ state was measured to be of the order of 50\% [9], however the precision of that measurement precludes quantitative conclusions about the suppression of the directly produced $\Upsilon(1 \mathrm{~S})$.

\section{Outlook}

Forthcoming p-A data will help to quantify cold nuclear matter effects and hopefully to sharpen the physics interpretation of the $\mathrm{Pb}-\mathrm{Pb}$ results.

The upgrades of the LHC experiments will improve significantly the capabilities to study HF production. For instance, the upgrade of the ALICE Inner Tracking system will allow ALICE to measure charm and beauty production in $\mathrm{Pb}-\mathrm{Pb}$ collisions with sufficient statistical accuracy down to very low transverse momentum, measure charm baryons and perform exclusive measurements of beauty production [9].

\section{References}

[1] M. Cacciari, M. Greco and P. Nason, JHEP 9805 (1998) 007;

M. Cacciari, S. Frixione and P. Nason, JHEP 0103 (2001) 006.

[2] D. Acosta et al. [CDF Coll.], Phys. Rev. D 71 (2005) 032001.

[3] M. Cacciari et al., JHEP 0407 (2004) 033.

[4] B.A. Kniehl et al., Phys. Rev. D 77 (2008) 014011.

[5] D. Acosta et al. [CDF Coll.], Phys. Rev. Lett. 91 (2003) 241804.

[6] M. Cacciari and P. Nason, JHEP 0309 (2003) 006.

[7] B.A. Kniehl et al., Phys. Rev. Lett. 96 (2006) 012001.

[8] A. Adare et al. [PHENIX Coll.], Phys. Rev. Lett. 97 (2006) 252002.

[9] B.I. Abelev et al. [STAR Coll.], Phys. Rev. Lett. 98 (2007) 192301;

W. Xie et al. [STAR Coll.], PoS(DIS2010)182 (2010).

[10] S. Alekhin et al., arXiv:hep-ph/0601013 (2006), chapter IV.

[11] N. Armesto, A. Dainese, C. A. Salgado and U. A. Wiedemann, Phys. Rev. D71 (2005) 054027.

[12] D. Acosta et al. (CDF Collaboration), Phys. Rev. D71 (2005) 032001.

[13] A. Abulencia et al. (CDF Collaboration), Phys. Rev. Lett. 99 (2007) 132001.

[14] S. Abachi et al. (D0 Collaboration), Phys. Lett. B370 (1996) 239.

[15] B. Abbott et al. (D0 Collaboration), Phys. Rev. Lett. 82 (1999) 35.

[16] A. Adare et al. (PHENIX Collaboration), Phys. Rev. Lett. 98 (2007) 232002.

[17] C.-H. Chang, Nucl. Phys. B 172 (1980) 425.

[18] R. Baier and R. Rückl, Z. Phys. C 19 (1983) 251.

[19] R. Baier and R. Rückl, Phys. Lett. B 102 (1981) 364.

[20] A. D. Frawley, T. Ullrich, and R. Vogt, Phys. Rept. 462 (2008) 125. 
[21] G. T. Bodwin, E. Braaten, and G. P. Lepage, Phys. Rev. D 51 (1995) 1125,

[22] P. Cho and A. K. Leibovich, Phys. Rev. D 53 (1996) 150.

[23] P. Cho and A. K. Leibovich, Phys. Rev. D 53 (1996) 6203.

[24] T. Matsui and H. Satz, Phys. Lett. B 178 (1986) 416.

[25] Z. Conesa del Valle et al., Nucl. 223 Phys. B 214 (2011) 3.

[26] A. Capella et al., Eur. Phys. J. C 58 (2008) 437.

[27] R. Vogt, Phys. Rev. C 81 (2010) 044903.

[28] R. Vogt, Phys. Rev. C 61 (2000) 035203.

[29] B. Alessandro et al. (NA50 Collaboration), Eur. Phys. J. C 39 (2005) 335.

[30] R. Arnaldi et al. (NA60 Collaboration), Phys. Rev. Lett. 96 (2007) 132302.

[31] A. Adare et al. (PHENIX Collaboration), Phys. Rev. C 84 (2011) 054912.

[32] L. Adamczyk et al. (STAR Collaboration), arXiv:1208.2736.

[33] N. Brambilla et al., Eur. Phys. J. C 71 (2011) 1534.

[34] X. Zhao and R. Rapp, Nucl. Phys. A 859 (2011) 114.

[35] Y. Liu, Z. Qu, N. Xu and P. Zhuang, Phys. Lett. B 678 (2009) 72.

[36] A. Andronic, P. Braun-Munzinger, K. Redlich and J. Stachel, J. Phys. G 38 (2011) 124081.

[37] H. van Hees, V. Greco, R. Rapp, Phys. Rev. C 73 (2006) 034913.

[38] D. Molnar, J. Phys. G 31 (2005) S421S428.

[39] B. Abelev et al. (ALICE Collaboration), JHEP 07 (2012) 191; arXiv:1205.4007.

[40] B. Abelev et al. (ALICE Collaboration), JHEP in press; arXiv:1205.5880.

[41] K. Aamodt et al. (ALICE Collaboration), JINST 3 (2008) S08002.

[42] G. Aad et al. (ATLAS Collaboration), JINST 3 (2008) S08003.

[43] S. Chatrchyan et al. (CMS Collaboration), JINST 3 (2008) S08004.

[44] A.A. Alves et al. (LHCb Collaboration), JINST 3 (2008) S08005.

[45] B. Abelev et al. (ALICE Collaboration), JHEP 1 (2012) 128.

[46] G. Aad et al. (ATLAS Collaboration), Phys. Lett. B 707 (2012) 438.

[47] B. Abelev et al (ALICE Collaboration), Phys. Lett. B 708 (2012) 265.

[48] B. Abelev et al. (ALICE Collaboration), arXiv:1205.5423.

[49] V. Khachatryan et al. (CMS Collaboration), Phys. Rev. Lett. 106 (2011) 112001.

[50] S. Chatrchyan et al. (CMS Collaboration), Phys. Rev. Lett. 106 (2011) 252001.

[51] S. Chatrchyan et al. (CMS Collaboration), Phys. Rev. D84 (2011) 052008.

[52] R. Aaij et al. (LHCb Collaboration), Phys. Lett. B 694 (2010) 209.

[53] V. Khachatryan et al. (CMS Collaboration), J. High Energy Phys. 3 (2011) 090.

[54] G. Aad et al. (ATLAS Collaboration), Nucl. Phys. B 850 (2011) 387.

[55] V. Khachatryan et al. (CMS Collaboration), Eur. Phys. J. C 71 (2011) 1575.

[56] S. Chatrchyan et al. (CMS Collaboration), JHEP 2 (2012) 011.

[57] R. Aaij et al.(LHCb Collaboration), Eur. Phys. J. C 71 (2011) 1645.

[58] M. Cacciari, S. Frixione, M.L. Mangano, P. Nason, and G. Ridolfi, J. High Energy Phys. 07 (2004) 033.

[59] M. Cacciari, S. Frixione, N. Houdeau, M.L. Mangano, P. Nason and G. Ridolfi, arXiv:1205.6344 (2012).

[60] K. Aamodt et al. (ALICE Collaboration), Pys. Lett. B 704 (2011) 442; ibidem Erratum.

[61] M. Butenschön and B.A. Kniehl, Phys. Rev. Lett. 106 (2011) 022003.

[62] Y.-Q. Ma, K. Wang, K.T. Chao, Phys. Rev. Lett. 106 (2011) 042002. 
[63] (ALICE Collaboration) Phys. Rev. Lett. 108 (2012) 082001.

[64] B. Gong and J.-X. Wang, Phys. Rev. D78 (2008) 074011.

[65] J.P. Lansberg, Eur. Phys. J. C 61 (2009) 693.

[66] B. Gong, X.Q. Li and J.-X. Wang, Phys. Lett. B673 (2009) 197.

[67] M. Butenschoen and B. A. Kniehl, Phys. Rev. Lett. 108 (2012) 172002; arXiv:1201.1872.

[68] R. Aaij et al. (LHCb Collaboration), arXiv:1204.1462.

[69] V. Khachatryan et al. (CMS Collaboration), Phys. Rev. D 83 (2011) 112004.

[70] G. Aad et al. (ATLAS Collaboration), Phys. Rev. Lett. 108 (2012) 152001.

[71] R. Aaij et al. (LHCb Collaboration), Eur. Phys. J. C 72 (2012) 2025

[72] R. Aaij et al. (LHCb Collaboration), Phys. Rev. Lett. 109 (2012) 172003.

[73] S. Chatrchyan et al. (CMS Collaboration), Phys. Rev. Lett. 108 (2012) 252002.

[74] R. Aaij et al. (LHCb Collaboration), Phys. Rev. Lett. 108 (2012) 251802.

[75] B. Abelev et al. (ALICE Collaboration), Phys. Lett.s B 712 (2012) 165-175.

[76] C. Blume, these proceedings.

[77] R. Aaij et al. (LHCb Collaboration), JHEP 06 (2012) 141.

[78] A. Berezhnoy, V. Kiselev, A. Likhoded and A. Onishchenko, Phys. Rev. D 57 (1998) 4385.

[79] J. Lansberg, Eur. Phys. J. C 61 (2009) 693.

[80] C. Kom, A. Kulesza and W. Stirling, Phys. Rev. Lett. 107 (2011) 082002.

[81] S. Baranov, A. Snigirev and N. Zotov, Phys. Lett. B 705 (2011) 116.

[82] S. Chatrchyan et al. (CMS Collaboration) Phys. Rev. Lett. 107 (2011) 052302.

[83] S. Chatrchyan et al. (CMS Collaboration), Phys. Rev, Lett. in press; arXiv:1208.2826.

[84] S. Adler et al. (PHENIX Collaboration), Phys. Rev. Lett. 96 (2006) 032301.

[85] A. Adare et al. (PHENIX Collaboration), Phys. Rev. C 84 (2011) 044905.

[86] B. Abelev et al. (STAR Collaboration), Phys. Rev. Lett. 98 (2007) 192301.

[87] N. Armesto, M. Cacciari, T. Hirano, J.L. Nagle and C.A. Salgado, J. Phys. G 37 (2010) 025104.

[88] B. Abelev et al (ALICE Collaboration), JHEP 09 (2012) 112.

[89] B. Abelev et al (ALICE Collaboration), Phys. Rev. Lett. 109 (2012) 112301.

[90] S. Chatrchyan et al. (CMS Collaboration), JHEP 05 (2012) 063.

[91] S. La Pointe et al. (ALICE Collaboration), these proceedings.

[92] Y. Liu, N. Xu and P. Zhuang, Nucl. Phys. A 834 (2010) 317c.

[93] T. Affolder et al. (CDF Collaboration), Phys. Rev. Lett. 84 (2000) 2094

[94] C. Kuhn et al. (ALICE Collaboration), these proceedings. 